\title{
KLIMA-, KLIMABODEN- UND KLIMAVEGETATIONSTYPEN
}

\section{Paul Schaufelberger}

Das Problem: Dass Klima, Boden und Vegetation in irgend einem bestimmten Zusammenhang stehen, steht wohl fest. Welches sind nun aber die natürlichen Klima-, Boden- und Vegetationstypen? Darüber gehen die Auffassungen noch heute auseinander und wir müssen feststellen, daß vor allem die Ansichten der Klimatologie, der Bodenkunde und der Botanik weit davon entfernt sind, sich zu decken. Wir stoßen vorerst noch auf unüberbrückbar scheinende Widersprüche. $\mathrm{Da}$ aber in jeder Auffassung ein Körnchen Wahrheit steckt, ist es vielleicht doch möglich, die verschiedenen Erfahrungen unter einen. Hut zu bringen.

Vorschlag von F.J. CALDAS. Der kolumbanische Naturforscher Franz Joseph Caldas (1771-1816) unterteilte das Tropenklima der südamerikanischen Anden nach den Jahrestemperaturen von $24,18,12$ und $6^{\circ} \mathrm{C}$ in fünf Höhengürtel und unterschied in jedem «sehr trockene», «trockene», «feuchte» und «sehr feuchte» Klimate. Jedem dieser 20 Klimatypen entspricht eine charakteristische landwirtschaftliche Nutzung. (Tabelle 1)

Tab. . . Tropenklimate und landwirtschaftliche Nutzung

\begin{tabular}{lllll}
\hline Höhenzone & sehr trocken & trocken & $\begin{array}{c}\text { Befeuchtung } \\
\text { feucht }\end{array}$ & sehr feucht \\
\hline $\begin{array}{l}\text { Tierra } \\
\text { caliente }\end{array}$ & Weide & $\begin{array}{l}\text { Baumwolle, Tabak } \\
\text { Mais }\end{array}$ & $\begin{array}{l}\text { Bananen, Kakao, } \\
\text { Zuckerrohr }\end{array}$ & Weide, Wald \\
\hline $\begin{array}{l}\text { Tierra } \\
\text { templada }\end{array}$ & Weide & $\begin{array}{l}\text { Tabak, Mais } \\
\text { Citrus }\end{array}$ & $\begin{array}{l}\text { Bananen, Kaffee, } \\
\text { Zuckerrohr }\end{array}$ & Weide, Wald \\
\hline $\begin{array}{l}\text { Tierra } \\
\text { fría }\end{array}$ & Weide & $\begin{array}{l}\text { Getreide, Mais, } \\
\text { Kartoffeln, Kern- } \\
\text { und Steinobst }\end{array}$ & Weide & Weide, Wald \\
\hline $\begin{array}{l}\text { Páramo } \\
\text { Tierra }\end{array}$ & Weide & Kartoffeln & Weide & Weide, Wald \\
helada & & & unkultivierbar & \\
\hline
\end{tabular}

Die Caldas'sche Klimasystematik basiert auf den Klimafaktoren: Zone, Jahrestemperatur und Befeuchtung. Für jeden Klimatyp wird auf Grund der Erfahrung die landwirtschaftliche Nutzung bestimmt. Diese üben die Andenbewohner seit anderthalb Jahrhunderten. $\mathrm{Da} ß$ sie wirklichkeitsgemäß ist, ergibt sich aus der Tatsache, daß sie trotz der verschiedenen Tropenklimate nirgends modifiziert werden mußte. Seit CALDAS besteht also mindestens für die Tropen eine praktisch brauchbare Klimasystematik.

Vorschlag von VILENSKy. Der bekannte russische Bodenkundler Vilensky (zitiert in H. JENny 1929) glaubt folgende Beziehungen zwischen Klima und Bodenbildung gefunden $\mathrm{zu}$ haben. (Tabelle 2)

Tab. 2. Klima und Bodenbildung

\begin{tabular}{|c|c|c|c|c|c|}
\hline Temperatur & arid & semiarid & $\begin{array}{l}\text { Befeuchtung } \\
\text { schwacharid }\end{array}$ & semihumid & humid \\
\hline $\begin{array}{l}\text { Polar } \\
-12 \text { bis } \\
-4^{\circ} \mathrm{C}\end{array}$ & $\begin{array}{l}\text { Tundra- } \\
\text { böden }\end{array}$ & $\begin{array}{l}\text { Semimoor- } \\
\text { böden }\end{array}$ & $\begin{array}{l}\text { Moor- und } \\
\text { Sumpfböden }\end{array}$ & - & $\begin{array}{l}\text { podsolierte } \\
\text { Böden }\end{array}$ \\
\hline $\begin{array}{l}\text { Kalt } \\
-4 \text { bis } \\
+4^{\circ} \mathrm{C}\end{array}$ & $\begin{array}{l}\text { Torf- } \\
\text { böden }\end{array}$ & 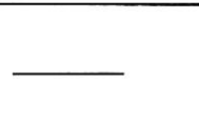 & $\begin{array}{l}\text { Schwarze } \\
\text { Wiesenböden }\end{array}$ & $\begin{array}{l}\text { degradierte } \\
\text { Wiesenböden }\end{array}$ & $\begin{array}{l}\text { podsolierte } \\
\text { Böden }\end{array}$ \\
\hline
\end{tabular}




\begin{tabular}{|c|c|c|c|c|c|}
\hline Temperatur & arid & semiarid & $\begin{array}{l}\text { Befeuchtung } \\
\text { schwacharid }\end{array}$ & semihumid & humid \\
\hline $\begin{array}{l}\text { Temperiert } \\
+4 \text { bis } \\
+12^{\circ} \mathrm{C}\end{array}$ & $\begin{array}{l}\text { Grau- } \\
\text { erden }\end{array}$ & $\begin{array}{l}\text { Kastanien- } \\
\text { böden }\end{array}$ & Tschernosem & $\begin{array}{l}\text { degradierte } \\
\text { Grauerden }\end{array}$ & $\begin{array}{l}\text { podsolierte } \\
\text { Böden }\end{array}$ \\
\hline $\begin{array}{l}\text { Subtropen } \\
+12 \text { bis } \\
+20^{\circ} \mathrm{C}\end{array}$ & 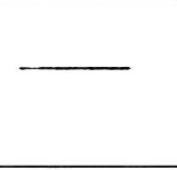 & $\begin{array}{l}\text { Gelberden } \\
\text { der ariden } \\
\text { Steppen }\end{array}$ & Gelberden & $\begin{array}{l}\text { degradierte } \\
\text { Gelberden }\end{array}$ & $\begin{array}{l}\text { podsolierte } \\
\text { Gelberden }\end{array}$ \\
\hline $\begin{array}{l}\text { Tropen } \\
\text { über } 20^{\circ} \mathrm{C}\end{array}$ & $\begin{array}{l}\text { Roterden } \\
\text { der Halb- } \\
\text { wüste }\end{array}$ & Roterden & Laterit & $\begin{array}{l}\text { degradierte } \\
\text { Roterden }\end{array}$ & $\begin{array}{l}\text { podsolierte } \\
\text { Roterden }\end{array}$ \\
\hline
\end{tabular}

VILENSKy benützt als Klimafaktoren fünf Befeuchtungsgrade und fünf Zonen, die er durch mittlere Jahrestemperaturen definiert. Dieser Vorschlag ist in der bodenkundlichen Literatur häufig zitiert und H. Jenny (1929) spendet ihm folgenden Beifall: «Jedem Bodentyp wird ein bestimmter Befeuchtungs- und Temperaturzahlenbereich zugeordnet. Interessant ist die Tatsache, daß im Zentrum des ganzen Systems der Tschernosem steht, der einem Klima entspricht, das nicht zu kalt und nicht zu warm, nicht zu trocken und nicht zu feucht ist.»

Vorschlag von W. LaUer. W. LAUER hält an der Auffassung der gemäßigten Zone fest, daß die Regenverteilung das Tropenklima bzw. die Befeuchtung bestimme, und auf dieser Grundlage entwirft er seine Isohygromenenkarte von Afrika und Südamerika. Je nach der Anzahl von humiden Monaten unterscheidet er trockene, wechselfeuchte und immerfeuchte Klimate und kombiniert dann diese mit den Höhenklimaten von Caldas. In jedem so erhaltenen Klimabereich vermutet er eine kennzeichnende tropische Vegetation, wie Tabelle 3 veranschaulicht.

Tab. 3. Horizontale und vertikale Klima- und Vegetationsgïrtel der tropischen Anden

\begin{tabular}{|c|c|c|c|c|}
\hline $\begin{array}{l}\text { Befeuchtung } \\
\text { humide Monate }\end{array}$ & Tierra caliente & $\begin{array}{l}\text { Temperatur } \\
\text { Tierra templada }\end{array}$ & Tierra fría & Tierra helada \\
\hline 12 bis 9 & $\begin{array}{l}\text { immergrüner } \\
\text { tropischer } \\
\text { Regenwald }\end{array}$ & $\begin{array}{l}\text { immergrüner } \\
\text { tropischer } \\
\text { Bergwald }\end{array}$ & $\begin{array}{l}\text { Immergrüner } \\
\text { tropischer } \\
\text { Höhen- und } \\
\text { Nebelwald }\end{array}$ & Páramo \\
\hline 9 bis 7 & $\begin{array}{l}\text { Feuchtsavanne. } \\
\text { regengrüner } \\
\text { Feuchtwald }\end{array}$ & $\begin{array}{l}\text { Feucht- } \\
\text { Valle }\end{array}$ & $\begin{array}{l}\text { Feucht- } \\
\text { Sierra }\end{array}$ & $\begin{array}{l}\text { Feucht- } \\
\text { puma } \\
\end{array}$ \\
\hline 6 bis 5 & $\begin{array}{l}\text { Trockensavanne. } \\
\text { regengrüner } \\
\text { Trockenwald }\end{array}$ & $\begin{array}{l}\text { Trocken- } \\
\text { Valle }\end{array}$ & $\begin{array}{l}\text { Trocken- } \\
\text { Sierra }\end{array}$ & $\begin{array}{l}\text { Trocken- } \\
\text { Puna }\end{array}$ \\
\hline 4 bis 2 & $\begin{array}{l}\text { Dornsavanne. } \\
\text { regengrüner } \\
\text { Dornwald }\end{array}$ & $\begin{array}{l}\text { Dorn- } \\
\text { Valle }\end{array}$ & $\begin{array}{l}\text { Dorn- } \\
\text { Sierra }\end{array}$ & $\begin{array}{l}\text { Dorn- } \\
\text { Puna }\end{array}$ \\
\hline 1 bis 0 & $\begin{array}{l}\text { Halbwüste } \\
\text { Wüste. }\end{array}$ & $\begin{array}{l}\text { Halbwüsten- } \\
\text { Valle. } \\
\text { Wüsten-Valle }\end{array}$ & $\begin{array}{l}\text { Wüsten- } \\
\text { Sierra }\end{array}$ & $\begin{array}{l}\text { Wüsten- } \\
\text { Puna }\end{array}$ \\
\hline
\end{tabular}

LAUER benützt als Klimafaktoren die Regenverteilung und die Höhenklimate von CaLDAS, aber ohne dessen Definition durch die Jahrestemperaturen von 24, 18, 12 und $6^{\circ} \mathrm{C}$ ! Die einzelnen Klimate definiert er dann durch hypothetische Vegetationstypen, was wohl kaum den Gesetzen der Logik entspricht. Aber der Beifall der gemäßigten Zone ist ihm sicher, wie aus einer brieflichen Mitteilung des Schweizerischen Tropen- 
institutes in Basel eindeutig hervorgeht: "Wenn Sie mit Recht kritisieren, daß man bei der Begriffsbildung der «Tropen» die horizontale Klimakomponente der tropischen Tiefebenen berücksichtige und dabei die vertikale Komponente vernachläßige, so sei doch darauf hingewiesen, daß diese Lücke seit langem ausgefüllt ist, nämlich seit man die Vegetation als Klimaindikator berücksichtigt und so den natürlichen Gegebenheiten besser gerecht wird.»

\section{DISKUSSION}

1. Caldas definiert seine Klimatypen durch die Befeuchtung, die Jahrestemperaturen von 24, 18, 12 und $6^{\circ} \mathrm{C}$ und die «Tropen», also die Zone. Genau definiert sind nur die Höhengürtel, während Zone und Befeuchtung gefühlsmäßig beurteilt werden. Aber für jeden Klimatyp läßt sich durch Erfahrung die landwirtschaftliche Nutzung bestimmen.

2. Vilensky kennt fünf Befeuchtungen und fünf Zonen, die durch die Jahrestemperaturen von $-12,-4,+4,+12$ und $+20^{\circ} \mathrm{C}$ definiert werden. Die Befeuchtung wird, wie bei Caldas, gefühlsmäßig beurteilt. In seinem Schema ist durch hypothetische Bodentypen gekennzeichnet, was wohl nicht ganz logisch ist. Daß dieser Vorschlag nicht mit den bekannten Tatsachen übereinstimmt, ergibt die Nachprüfung. Z.B. läßt sich fragen: 1. Warum bilden sich die Gelberden der ariden Steppen ausgerechnet im semiariden subtropischen Klima? 2. Sind die Niedermoore tatsächlich auf das aride kalte (d.h. subpolare Gebiet) beschränkt? 3. Kommen Semimoorböden (Anmoore) wirklich nur im Polargebiet vor?

H. JENNy behauptet, daß im Vorschlag von VILENSKy jeder Bodentyp durch «einen bestimmten Befeuchtungs- und Temperaturzahlenbereich» begrenzt sei. Durch welche Zahlenbereiche VILENSKY's sind indes «aride», «semiaride», «schwacharide», «semihumide» und «humide» Befeuchtungen begrenzt?

3. LAUER definiert die Klimate nach der Regenverteilung durch hypothetische Vegetationstypen! Betrachten wir einmal seine Angaben für die tierra caliente! Hier finden wir zwei Reihen ineinandergeschachtelt:

1. Halbwüste, Dornsavanne, Trockensavanne, Feuchtsavanne und Regenwald.

2. Dornwald, Trockenwald, Feuchtwald und Regenwald.

Darf man das Schweizerische Tropeninstitut in Basel fragen, welche dieser beiden Vegetationstypen nun die tatsächlichen Klimaindikatoren sind? Nach den Behauptungen der gemäßigten Zone wären Steppe, Savanne und Regenwald die klimabedingten Vegetationen der Tropen, nach den Erfahrungen der Bodenkunde wären sie edaphisch bestimmt. Gewiß finden wir den ersten Irrtum in der Literatur sehr häufig, aber die Geschichte der Wissenschaften kennt keinen einzigen Fall, daß ein Irrtum durch noch so häufige Wiederholung zur Wahrheit geworden wäre!

Die zweite Reihe entspricht auch den Erfahrungen der Tropen, wo man seit langem folgende Beziehungen zwischen Klima und Wald kennt: arid: Trocken- und Dornwald ; semiarid: Monsunwald; semihumid: Laubwald, humid: Mischwald und perhumid: Palmenwald.

Wenn auch keiner der drei erwähnten Vorschläge die Klimasystematik gelöst hat, so stimmen sie doch darin überein, daß Zone, Jahrestemperatur und Befeuchtung eine maßgebende Rolle spielen. Aber wie sind sie zu definieren bzw. zu deuten?

A. Befeuchtung. Wie soll man die Befeuchtung definieren? a) nach der Regenmenge? b) nach der absoluten, c) nach der relativen Luftfeuchtigkeit? d) nach Niederschlag und Verdunstung? e) nach dem Regenfaktor? f) nach dem Ariditätsindex? g) nach den $N-S$-Quotienten? h) nach Klimabodentypen; i) nach Vegetationstypen? oder k) nach dem Gefühl? 
Wohl ist man sich darin einig, daß Jahrestemperatur und Jahresniederschlag zwei Großklimawerte darstellen und darum auch seit langem gemessen werden. Es ist das Verdienst von R. LANG diese beiden Faktoren zur graphischen Klimadarstellung benützt zu haben, indem er die Regenmenge auf der Abszisse, die Temperatur auf der Ordinate abträgt. Dieses Klimafeld unterteilt er durch die Regenfaktoren = Jahresniederschlag: Jahrestemperatur von 40,60, 100 und 160 und erhält so fünf wohl definierte Teilfelder: arid, semiarid, semihumid, humid und perhumid. Figur 1.

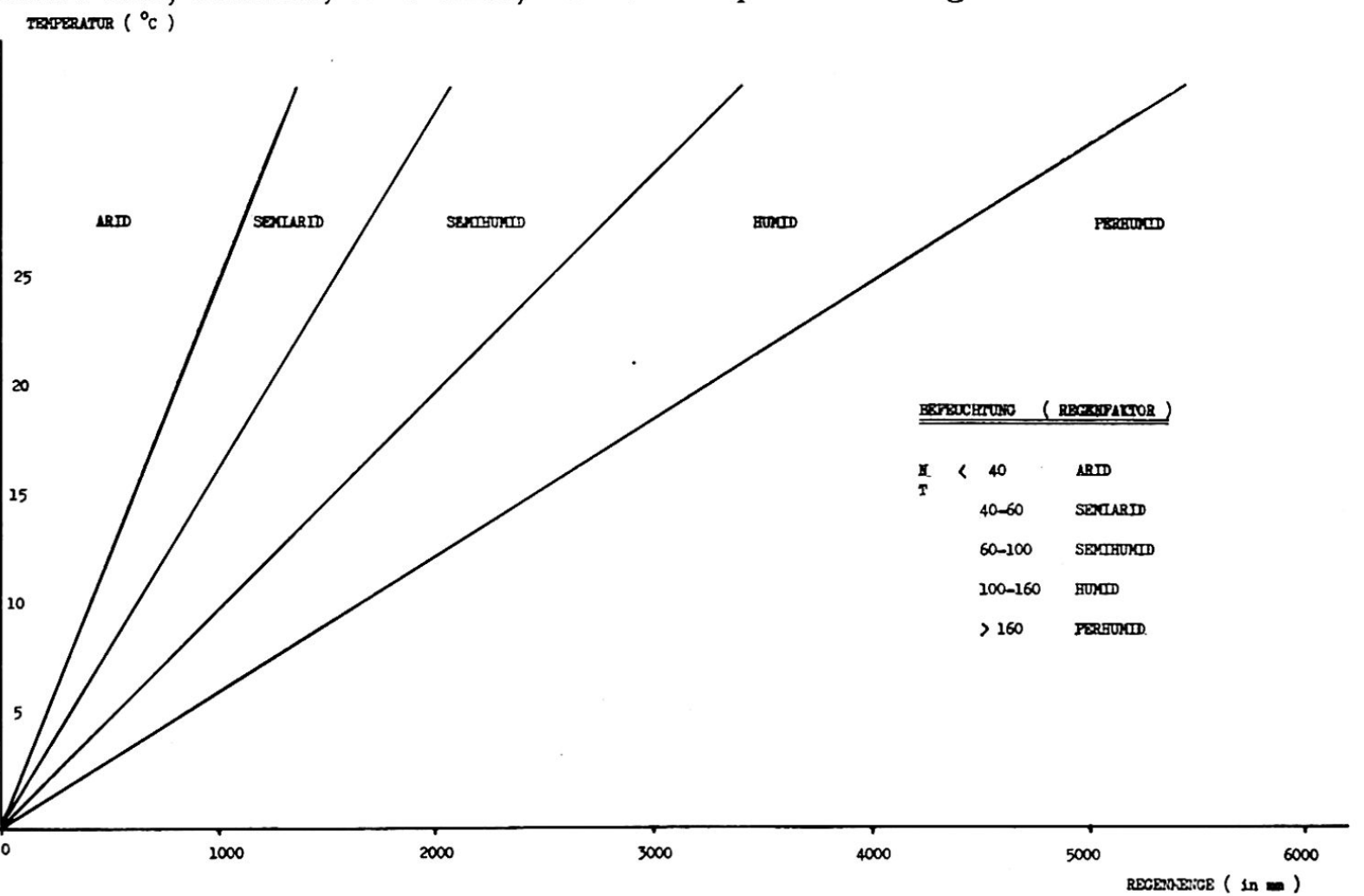

Figur 1 Regenfaktor von R. LANG

Damit sind schon die lange gebrauchten Bezeichnungen für die Befeuchtung eindeutig definiert. Allerdings lehnen Meteorologen, Klimatologen und teilweise auch Bodenkundler den Regenfaktor gefühlsmäßig als unbrauchbar für die Klimatologie ab; aber so lange sie nicht etwas Besseres als Ersatz zu bieten haben, werden sie schon gestatten müssen, daß wir ihn weiter benützen.

a) Im Vorschlag von CALDAs lassen sich «sehr trocken», «trocken», «feucht» und «sehr feucht» ohne weitere Schwierigkeiten durch die Regenfaktoren von 40, 60 und 100 definieren! Durch den Regenfaktor 160 wird «sehr feucht» in humid und perhumid weiter unterteilt, ohne daß dadurch die Klimasystematik von CaLDAs prinzipiell geändert wird!

b) VILENSKy's aride, semiaride, schwacharide, semihumide und humide Bereiche lassen sich ebenfalls ohne weiteres durch die Regenfaktoren von 40, 60, 100 und 160 definieren.

c) LAUER nennt edaphisch und klimatisch bedingte Vegetationstypen. $\mathrm{Zu}$ diesen gehören seine Trocken-, Feucht- und Regenwälder, die mit der Befeuchtung den Baumbestand wechseln. Nach einer brieflichen Mitteilung Herrn Dr. W. LAUER's entwickelt sich der «Regenwald der immerfeuchten Tropen» bei Jahresregenmengen zwischen 140 und $250 \mathrm{~cm}$. Das bedeutet für die tierra caliente ein semihumides Klima, das in den innern Tropen zwei Regenmaxima aufweist, wobei alle Monate humid sind; dort ist das semihumide Klima immerfeucht; in den äußern Tropen zeigt das selbe Klima eine Regenzeit, und die Trockenzeit ist eine Trockenperiode mit rund 6 ariden Monaten: dasselbe Klima ist wechselfeucht! 
Definieren wir die Befeuchtung durch die Regenfaktoren von R. LANG, dann ergeben sich folgende Übereinstimmungen der Tatsachen, wie Tabelle 4 zeigt.

Tab. 4. Regenfaktor und Befeuchtung von Caldas, Vilensky und Lauer

\begin{tabular}{lllllll}
\hline $\begin{array}{l}\text { Höhengürtel: } \\
\text { Tierra caliente. }\end{array}$ & & \multicolumn{2}{c}{ Befeuchtung $=$ Regenfaktor } \\
\hline CALDAS & sehr trocken & trocken & feucht & sehr feucht & sehr feucht \\
\hline LANG & arid & semiarid & semihumid & humid & perhumid \\
\hline VILENSKY & arid & semiarid & schwacharid & semihumid & humid \\
\hline LAUER & Trockenwald & Feuchtwald & Regenwald & $?$ & $?$ \\
\hline $\begin{array}{l}\text { SCHAUFELBERGER } \\
(1952)\end{array}$ & $\begin{array}{l}\text { Trockenwald } \\
\text { regengrün }\end{array}$ & $\begin{array}{l}\text { Monsunwald } \\
\text { regengrün }\end{array}$ & $\begin{array}{l}\text { Laubwald } \\
\text { immergrün }\end{array}$ & $\begin{array}{l}\text { Mischwald } \\
\text { immergrün }\end{array}$ & $\begin{array}{l}\text { Palmenwald } \\
\text { immergrün }\end{array}$ \\
\hline
\end{tabular}

Nun zeigt sich, daß in der tropischen tierra caliente die Befeuchtung die floristische Zusammensetzung der Wälder bestimmt, aber auch, daß LAUER die Tatsachen der humiden und perhumiden Tropen nicht kennt! Die abgelehnten Regenfaktoren bringen die Lücke an den Tag! Wir können sie ausfüllen.

B. Jahrestemperatur. VILENSKy definiert durch sie die Zonen, während CALdAS sie zur Charakterisierung der Höhengürtel benützt. Für die Tropen verwendet er diejenigen von $24,18,12$ und $6^{\circ} \mathrm{C}$. Für die gemäßigte Zone nennen GracaNiN, BacH und Deuel die Temperaturen von $4,8,12$ und $16^{\circ} \mathrm{C}$, die mit den von Vilensky und JENNY benützten Jahrestemperaturen gut übereinstimmen. Offenbar kennzeichnen die Jahrestemperaturen von $4,8,12$ und $16^{\circ} \mathrm{C}$ die Grenzen der Höhenklimate der gemäßigten Zone und nicht Zonengrenzen. Wollen wir an den Zonengrenzen Sprünge vermeiden, so können wir die kennzeichnenden Temperaturen der beiden Zonen miteinander verbinden, und diese Höhengrenzen unterteilen dann das Klimafeld vom Pol bis zum Aequator in fünf Höhengürtel, deren Grenzen sich, wie Figur 2 zeigt, 117 Breitegrade vom Aequator schneiden. Die geographische Breite $\varphi$ bestimmt dann die Zone und die Temperatur $\mathrm{T}$ das zonale Höhenklima.

Algebraisch ergeben sich dann für die fünf Höhengürtel nachstehende Definitionen:

$\begin{array}{lr}\text { nivale Klimate }: T:(117-\varphi) & <0,055 \\ \text { alpine Klimate } & 0,055 \text { bis } 0,110 \\ \text { montane Klimate } & 0,110 \text { bis } 0,165 \\ \text { colline Klimate } & 0,165 \text { bis } 0,220 \\ \text { marine Klimate } & >0,220\end{array}$

Damit sind die Höhenklimate durch die geographische Breite $\varphi$ und die Jahrestemperatur $T$ ebenfalls zahlenmäßig definiert.

C. Zone. Für die Abgrenzung der geographischen Zonen bestehen folgende Vorschläge: a) durch $W$ ende- und Polarkreise; b) durch die Breiten von 18, 36, 54 und $72^{\circ}$ lassen sich Tropen, Subtropen, gemäßigte Zone, Subpolar- und Polargebiete definieren; c) durch Jahresisothermen und d) durch das Gefühl. Daß die Tropen nicht durch Jahresisothermen als heiße Zone mit Jahrestemperaturen von über $20^{\circ} \mathrm{C}$ definieren lassen, dürfte heute auch den Klimatologen und Meteorologen der gemäßigten Zone bekannt sein. Es bleibt dann nur noch die Frage zu lösen, ob man drei oder fünf klimatische Zonen wählen will. Praktisch hat sich wohl die letztere Einteilung bewährt, die auch VILENSKy benützt, so daß wir die Klimazonen durch die Wendekreise von $18,36,54$ und $72^{\circ}$ begrenzen. 
D. Klimabodentypen. In VILENSKY's Schema finden wir als Klimabodentypen für die gemäßigte Zone: 1. Grauerden, 2. Kastanienböden, 3. Tschernosem, 4. degradierte Grauerden und 5. podsolierte Böden. Schon H. JENNy (1928) erbrachte den Nachweis, daß Böden auf kalkführenden Gesteinen keine echten Klimaböden seien. Zu diesen Böden gehören, wie derselbe Autor 1929 zeigte, die Grauerden (graue Wüstenböden auf Löß=Sierosem) und Kastanienböden. Nach VILENSKy sollte sich der Tschernosem bei Regenfaktoren zwischen 60 und 100 und Temperaturen zwschen +4 und $+12^{\circ} \mathrm{C}$ bilden, aber H. JENNy (1929) erbrachte den Nachweis, daß dieser Boden sich im gemäßigten Klima bei Regenfaktoren zwischen 40 und 150 und Temperaturen zwischen 2,8 und $13^{\circ} \mathrm{C}$ entwickelt. Der Tschernosem ist also ganz sicher kein Klimabodentyp, was Verf. auch an anderer Stelle bewies. Nach Vilensky bilden sich podsolierte Böden im perhumiden Klima in allen Zonen, was mit der Annahme LaNG's übereinstimmt, wonach die Klimaböden ganz unabhängig von der Zone durch die Befeuchtung bestimmt sind.

In Kolumbien wurden die Klimaböden mit Hilfe der Regenfaktoren gesucht und gefunden. An anderer Stelle (Schaufelberger 1950, 1952, 1958) sind sie als Kaktus-, Bambus-, Humus-, Wald- und Urwaldsols beschrieben und definiert. Zu den letzteren gehört der Podsol LANG's und Vilensky's, während die übrigen in der bodenkundlichen Literatur nicht als Klimaböden bekannt sind. Es sind basenarme, normal drainierte Waldböden mit ABC-Profilen (Oberboden, Unterboden und Muttergestein).

Daß diese Böden auch in Europa gefunden wurden, geht eindeutig aus der Beschreibung von H. Franz hervor: «Der sol lessivé (lessivé = ausgelaugt, ausgewaschen) der Franzosen und Belgier (Synonyme: grey-brown-podsolic soil der Amerikaner und Parabraunerde MückenhaUsen's und anderer deutscher Bodenkundler) ... ist ein Boden mit geringer Basensättigung trotz mehr oder weniger reichlichen Vorhandenseins von Tonmineralien. Es ist stets eine gewisse, wenn auch nur teilweise Ton- und Eisenverlagerung aus dem Eluvialhorizont in den Illuvialhorizont festzustellen, der Boden besitzt demnach ein ABC-Profil. - Sols lessivés wurden unter diesem Namen aus Nord- und Westfrankreich beschrieben und auch in Belgien in weiter Verbreitung nachgewiesen. Sie finden sich dort hauptsächlich auf Löß und kommen auf diesem auch in Westdeutschland vor. Im Nordosten der USA haben sie südlich der großen Seen eine große Verbreitung und sind dort unter dem Namen grey-brownpodsolic soils beschrieben worden. In Österreich finden sich sols lessivés im gesamten nördlichen Alpenvorland ostwärts bis zum Wienerwald auf Löß und vielleicht auf Schlier, ferner in der Flyschzone und auf Flyschgestein und schließlich auf silikatischen Gesteinen im gemäßigt humiden Klima. - Die Entwicklung eines sol lessivé zeigt stets drei Dinge an: eine geringe Basensättigung des Bodens, relative Dichtelagerung und ein starkes Vorherrschen der Sickerwasserbewegung.»

Damit ist der Beweis erbracht, daß sich neben den basenreichen Waldböden: Sierosem, Kastanienboden, Braunerden, Rendzina, Humuskarbonatboden, Schwarzerden, terra rossa, tierra roxa usw., die nach JENNy (1928) keine echten Klimaböden sind, noch basenarme Waldböden (sols lessivés) bilden, die dem Klima entsprechen.

Die von LANG vermuteten fünf Klimabodentypen sind basenarme Waldböden, die mit der Befeuchtung den Humusgehalt und Chemismus wechseln.

Zusammenhang zwischen Klima, Bodenbildung und Vegetation. Definieren wir in VILENSKy's Vorschlag die Zonen durch die Breitegrade 72, 54, 36 und 18, die zonalen Höhenklimate durch die Jahrestemperaturen : 2, 4, 6 und $8^{\circ}$ (Polargebiet); bzw. 3, 6, 9 und $12^{\circ}$ (Subpolarzone) ; 4, 8, 12 und $16^{\circ} \mathrm{C}$ (gemäßigte Zone); bzw. 5, 10, 15 und $20^{\circ} \mathrm{C}$ (Subtropen) ; bzw. 6, 12, 18 und $24^{\circ} \mathrm{C}$ (Tropen) und die Befeuchtungen durch die Regenfaktoren 40, 60, 100 und 160, so ergibt sich zwischen Pol und Aequa- 
tor folgende Klimasystematik. Tabelle 5. Dieser fügen wir noch die Klimabodentypen und die Waldvegetation für die tropische tierra caliente oder das tropische marine Klima bei.

Tab. 5. Klimasystematik Caldas-Lang-Vilensky

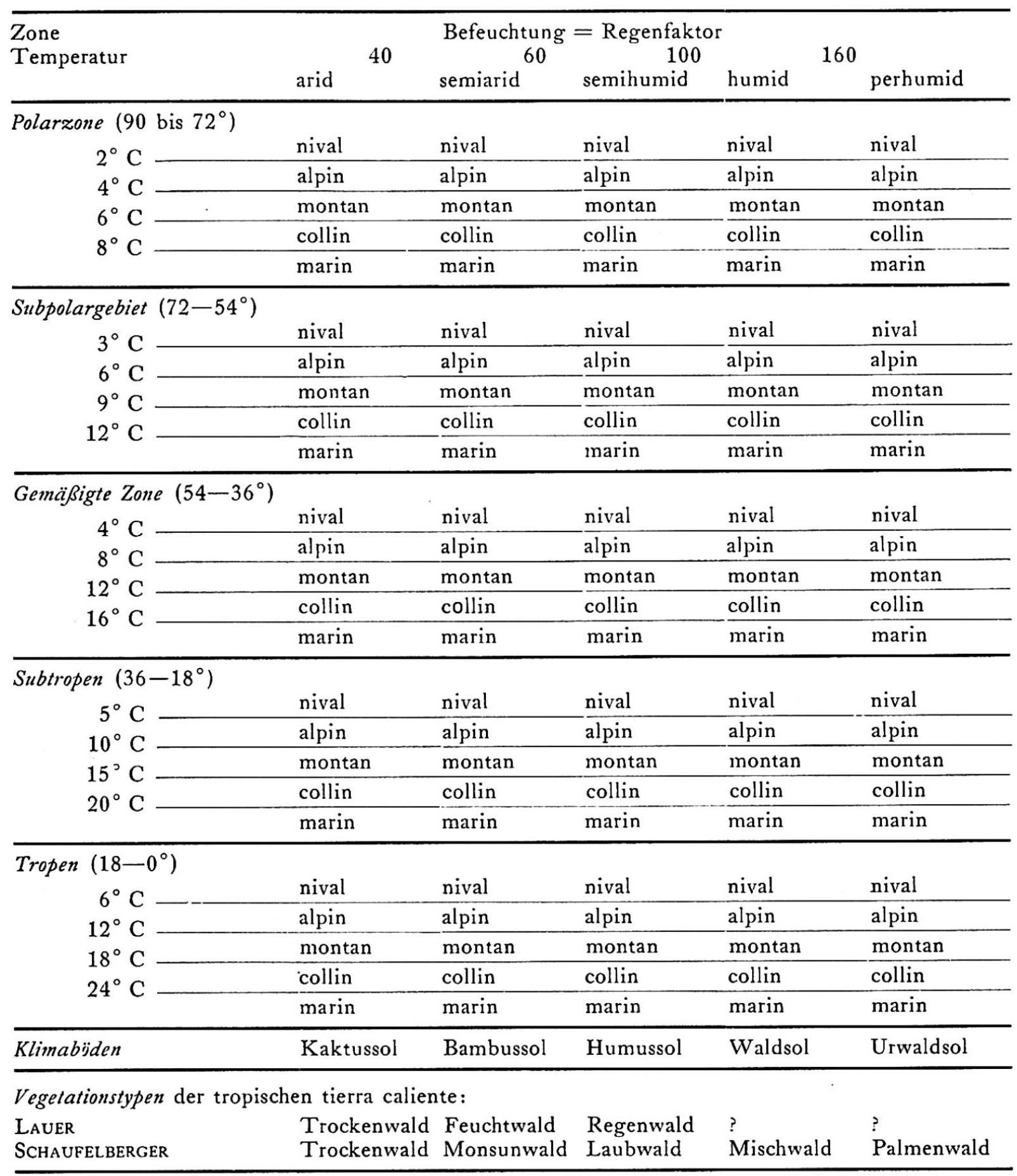

\section{ZUSAMMENFASS UNG}

Es sind zwei unabhängige Klimafelder zu unterscheiden: 1. Trägt man die Regenmenge auf der Abszisse und die Temperatur auf der Ordinate ab, dann wird dieses Klimafeld durch die Regenfaktoren 40,60, 100 und 160 in die Befeuchtungsfelder arid, semiarid, semihumid, humid und perhumid unterteilt. 2. Trägt man auf der Grundlinie die geographische Breite und die Temperatur auf der Ordinate ab, dann 
wird dieses Feld durch die Breiten von 18, 36, 54 und 72 in 5 Zonenklimate und durch die Höhengrenzen in je fünf Zonenhöhenklimate unterteilt. Die letztern sind durch die geographische Breite und durch die Jahrestemperatur bestimmt. Fig. 2. In jedem dieser Höhenklimate sind dann 5 Befeuchtungen, Fig. 1, zu unterscheiden.

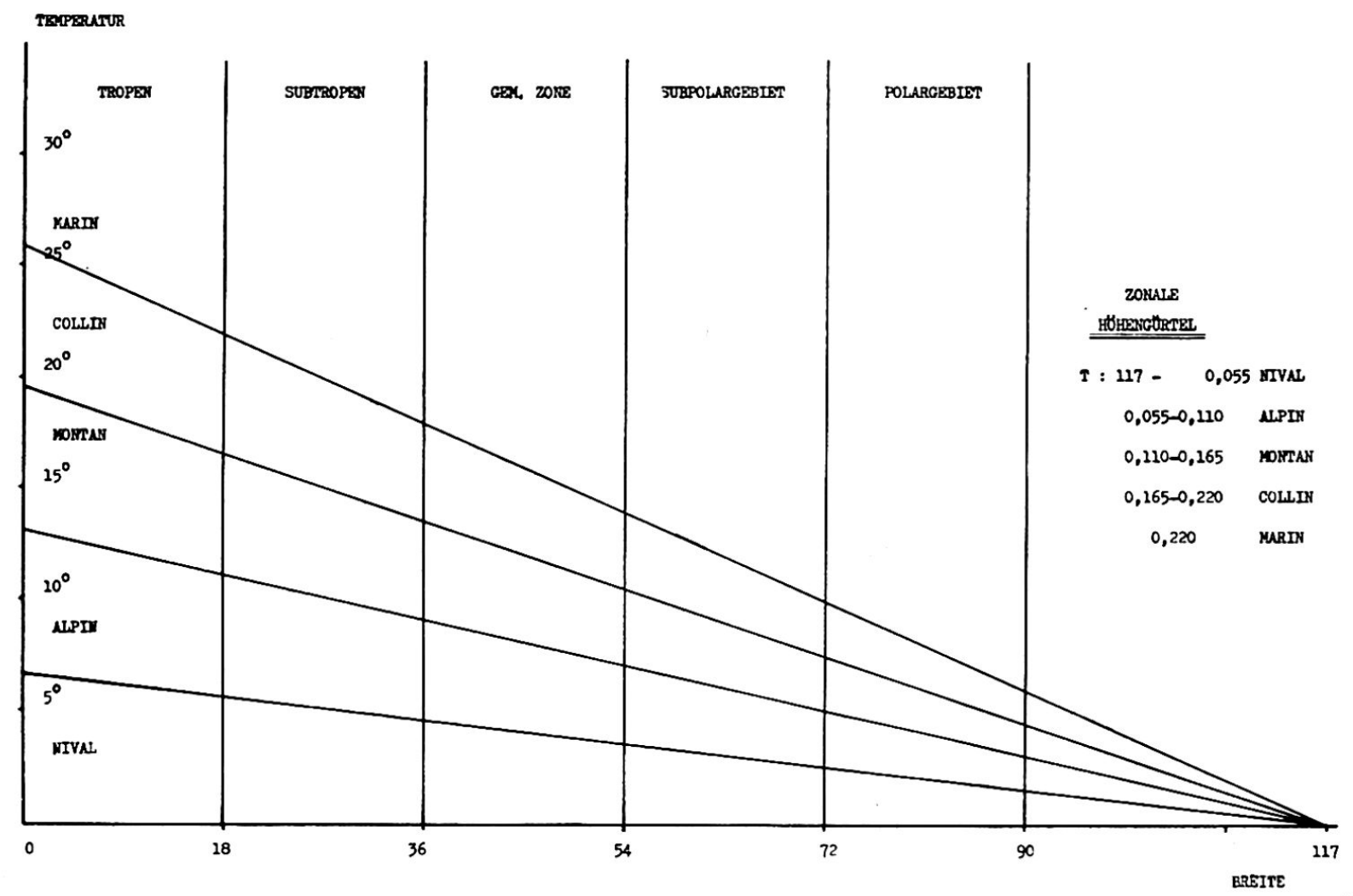

Lies: $\mathrm{T}:(117-\varphi):<0,055 ;>0,220$

Figur 2 Zonale Höhengürtel

\section{Zone, Jahrestemperatur und Befeuchtung definieren dann das Lokalklima.}

Die Klimabodentypen sind normal drainierte, basenarme Waldböden, sols lessivés, deren Humusgehalt und Chemismus durch die Befeuchtung bestimmt wird.

Die natürliche Vegetation dieser Klimaböden ist ein Wald, dessen floristische $\mathrm{Zu}$ sammensetzung noch durch Zone und Jahrestemperatur bestimmt wird. (Wiesen und Savannen, Steppen, Salzsteppen, Anmoore und Moore sind edaphisch bestimmt und weitgehend aklimatisch, sie sind also keine Klimaindikatoren, trotzdem dies leider in der Literatur vielfach behauptet wird.)

\section{EINIGE LITERATURHINWEISE}

1. BACH, R. und Devel, H.: Polykopien zur Vorlesung über Bodenkunde an der ETH Zürich. 2. Caldas, F. J.: Estudios seleccionados referentes a Astronomía, Meteorología y Física. Rev. de la Academía Colombiana de Ciencias Exactas, Física y Naturales II/6 (1938). - 3. Franz, H.: Bodenkunde. Hochschule für Bodenkultur Wien. - 4. Gracanin, M.: Monthly Rain-factors and their Significance for pedological Investigations. Revisio Scientifica Agriculturae 12, p. 51-61 (1950). 5. Jenny, H.: Bemerkungen zur Bodentypenkarte der Schweiz. Schweiz. Landw. Jahrb. 42/3 (1928). 6. JenNy, H.: Klima und Klimabodentypen in Europa und den Vereinigten Staaten von Nordamerika. Bodenkundl. Forschungen I/3 (1929). - 7. LANG, R.: Versuch einer exakten Klassifikation der Böden in klimatischer und geologischer Hinsicht. Int. Mitt. f. Bodenkunde 1915. - 8. LAUER, W.: Humide und aride Jahreszeiten in Afrika und Südamerika und ihre Beziehungen zu den Vegetationsgürteln. Bonner Geogr. Abhandlg. Heft 9 (1952). - 9. Schaufelberger, P.: Wie verläuft die Gesteinsverwitterung und Bodenbildung in den Tropen, insbesondere in Kolumbien? Schweiz. Min. u. Petr. Mitt. 30/1 (1950). - 10. Schaufelberger, P.: Die Klimabodentypen des tropischen Kolumbiens. Vierteljahrsschr. d. Naturf. Ges. in Zürich 97, p. 92-114 (1952a). - 11. Schaufelberger, P.: Zur Gliederung des tropischen Klimas. Geogr. Helv. VII/2 (1952 b). - 12. Schaufelberger, P.: Gibt es 
Tschernosem in den Tropen? Zeitschr. f. Pflanzenern., Düngung u. Bodenkunde 57, p. 121-134 (1952 c). - 13. Schaufelberger, P.: Zur Systematik des Tropenklimas. Geogr. Helv. XII/1 (1957). 14. Schaufelberger, P.: Warum haben die Regenfaktoren so häufig in Klimatologie und Bodenkunde versagt? Ibid. XIII/1 (1958a). - 15. SchaufelBerger, P.: Welches sind nach den Erfahrungen der Bodenkunde die maßgebenden Klimafaktoren? Petermanns Geogr. Mitt. 2. Quartalsheft (1958b). 16. Schaufelberger, P.: Die Bodentypen des modifizierten Systems Pallmann. Schweiz. Min. u. Petr. Mitt. 38/1 (1958 c) - 17. Mapas climatológicos para Colombia y Costa Rica. Cenicafé 9/3-4 (1958 d. 18. VILENSKY, zitiert in H. JENNY 1929.

\section{TYPES DE CLIMATS ET TYPES DE SOLS ET DE VÉGÉTATIONS CLIMATIQUES}

Il faut distinguer deux champs climatiques indépendants l'un de l'autre: 1 . Si l'on reporte les quantités de pluie en abscisses et les températures en ordonnées, les facteurs de pluie 4o, 60 , 100 et 160 subdivisent le premier champ climatique en zones d'humidification aride, semiaride, semi-humide, humide et très humide. 2. Lorsque l'on reporte les latitudes sur l'axe des abscisses et les températures sur celui des ordonnées, les latitudes 18, 36, 54 et 72 subdivisent ce second champ en 5 zones climatiques horizontales et les limites d'altitude en 5 zones climatiques verticales. (Fig. 2). Ces dernières sont déterminées par la latitude et la température annuelle. Dans chacun de ces climats en hauteur, on distingue cinq processus d'humidification. $L a$ zone, la température annuelle et l'humidification définissent donc le climat local. Les types de sols climatiques sont des sols forestiers normalement drainés, pauvres en bases, des «sols lessivés», dont la teneur en humus et le chimisme sont déterminés par l'humidification. La végétation naturelle de ces sols climatiques est une forêt, dont l'association végétale est une fois encore déterminée par la zone à laquelle elle appartient et la température annuelle. (Les prairies. savanes, steppes, steppes salées, marécages et régions avoisinantes - «Anmoore» - sont déterminés pédologiquement et le plus souvent sans rapport avec le climat; ils sont donc de mauvais indicateurs climatiques, en dépit de ce que l'on prétend trop souvent dans les ouvrages spécialisés).

\section{DIE SCHWEIZ 1958}

\section{EIN LANDESKUNDLICHER RÜCKBLICK}

Das Jahr 1958 brachte der Schweiz einen gesamtlandschaftlich folgenreichen Volksentscheid. Am 6. Juli wurde ein Verfassungsartikel verabschiedet, nach welchem der «Ausbau eines schweizerischen Straßennetzes» und insbesondere eines Autobahnnetzes zu fördern ist». Die Projektierungen ( $571 \mathrm{~km}$ Nationalstraßen I., $559 \mathrm{~km}$ II. und $542 \mathrm{~km}$ III. Klasse sowie $36 \mathrm{~km}$ Expreß- und Stadtstraßen) lassen erkennen, da $\beta$ das Werk unmittelbar und mittelbar große Auswirkungen auf Siedlung und Wirtschaft haben wird. Der Landes- und Landschaftsplanung und Landschaftsgestaltung im weitern Sinne erwachsen hieraus komplexe Aufgaben, welche in den kommenden Jahren Fachleute und Öffentlichkeit in zunehmendem Maße beschäftigen werden. Die übrigen Vorgänge hielten sich mehr im Rahmen der Vorjahre.

Naturereignisse. Bodenbewegungen: Nach einer Statistik des Geologen Dr. H. StaUber ereigneten sich 195824 bemerkenswerte Rutsche, Bergstürze und andere Bodenbewegungen, wovon 14 auf die Alpen (Wallis, Berner Oberland, Gotthard, Graubünden, Glarus-Uri, Tessin), 7 auf das Mittelland (Appenzell, Zürcher Oberland, Zug, Entlebuch) und 3 auf den Jura (Önsingen, Moutier, Biel) entfallen. Besonders interessant waren die Eisabbrüche vom Gspaltenhorn-Gletscher ins Sefinental im August. Gegenüber den vorangehenden 5 Jahren, während denen im Mittel je rund 8o Fälle gemeldet wurden, war 1958 somit eher ein «ruhiges» Jahr, wenn auch einzelne Vorkommnisse wie namentlich die seit längerem anhaltende Schuderser Rutschung bedenkenerregend waren.

Für den Witterungsgang war, wie Dr. M. ScнüEPP von der meteorolog. Zentralanstalt mitteilt, charakteristisch, da $\beta$ die Alpennordseite einen Temperaturüberschu $\beta$ von etwa $1 / 2^{-1}{ }^{\circ}$ gegenüber dem Normalwert hatte, sodaß 1958 wie 1957 zu den warmen Jahren gezählt werden kann. Südlich der Alpen wurden etwas kleinere positive Abweichungen von einigen Zehntelsgraden gemessen. Größere positive Abweichungen brachten vor allem die Monate Februar, Mai, August, September und Dezember, während das spätere Frühjahr (März-April) wesentlich zu kalt ausfiel, sodaß ein bedeutender Rückstand in der Vegetation eintrat.

Die Niederschläge blieben im Jura und am Juranordfuß um etwa $10 \%$ unter der langjährigen Jahressumme, während sie diese in den übrigen Teilen der Schweiz größtenteils überschritten. In den meisten Gegenden betragen die Überschüsse $5-10 \%$, in den Alpen jedoch teilweise 15-25\% . Den Hauptanteil des Überschusses lieferte der sehr niederschlagsreiche Februar, nördlich der Alpen auch der Januar. Ferner waren beidseits der Alpen August und Oktober strichweise niederschlagsreich. Auf der Alpensüdseite brachte der Dezember große Regen- und 